\title{
Impacto de los influencer en los estudiantes de la Facultad de Ciencias Empresariales de la Universidad Técnica Estatal de Quevedo.@()@(@)
}

\section{Impact of influencers on students of the Faculty of Busindess Sciences of the State Technical University of Quevedo.}

José Fabián Fonseca Vasconez ${ }^{1}$, Erika Yessenia Ballesteros Ballesteros ${ }^{2}$, \& Hugo Andrés Vinueza Peralta ${ }^{3}$

Recibido: 17-12-2020 / Revisado: 07-01-2021 /Aceptado: 14-01-2021/ Publicado: 05-02-2021

\begin{abstract}
:
DOI: https://doi.org/10.33262/concienciadigital.v4i1.1.1559

Introduction: the knowledge society imposes a paradigm shift in the training of professionals, considering that the occupational needs in the different sectors of the administration of productive operations and services, where the use of information and communication technologies, transform the dynamics of the Results, laws and essential relationships between objects and phenomena of reality. Objective: to identify the role and impact of the figure of the influencer in the training of students of the Faculty of Business Sciences of the State Technical University of Quevedo. Methodology: it is based on methods of theoretical and empirical level; in this sense. Results: for the sake of deepening this topic, a compilation of information has been made about the phenomenon of influencers, its evolution, features and characteristics, classifications, as well as the qualities of influences that have occurred in the area of management knowledge. commercial, economic, financial and accounting Conclusion: it is determined that the influencers have communication strategy for brand positioning, which has obtained great relevance in the 2.0 panorama in a short period of time
\end{abstract}

Key words: Influence, prescribers, influencers, social networks, students

\section{Resumen}

Introducción: la sociedad del conocimiento impone un cambio de paradigmas en la formación de los profesionales, en consideración que las necesidades ocupacionales en

\footnotetext{
${ }^{1}$ Universidad Técnica Estatal de Quevedo, Facultad de Ciencias Empresariales, Quevedo, Ecuador, jfonsecav@uteq.edu.ec, https://orcid.org/0000-0003-3975-8751

${ }^{2}$ Universidad Técnica Estatal de Quevedo, Facultad de Ciencias Empresariales, Quevedo, Ecuador, eballesteros@uteq.edu.ec, https://orcid.org/0000-0001-5601-5541

${ }^{3}$ Universidad Técnica Estatal de Quevedo, Facultad de Ciencias Empresariales, Quevedo, Ecuador, hvinuezap@uteq.edu.ec, https://orcid.org/0000-0001-7771-843
} 
los diferentes sectores de la administración de operaciones productivas y de servicios, donde el empleo de las tecnologías de la información y las comunicaciones, transforman la dinámica de las Resultados leyes y relaciones esenciales entre los objetos y fenómenos de la realidad. Objetivo: identificar el papel e impacto que ejerce la figura del influencer en la formación de estudiantes de la Facultad de Ciencias Empresariales de la Universidad Técnica Estatal de Quevedo. Metodología: se fundamenta, en métodos de nivel teórico, empírico; en este sentido. Resultados: en aras de la profundización en este tema se ha realizado una recopilación de información acerca del fenómeno de los influencers, su evolución, rasgos y características, clasificaciones, así como las cualidades de influencias que han sucedido en el área del conocimiento de la gestión comercial, económica, financiera y contable Conclusión: se determina que los influencer tienen como estrategia la comunicación para el posicionamiento de marcas, la que ha obtenido una gran relevancia en el panorama 2.0 en un periodo corto de tiempo.

Palabras clave: Influencia, prescriptores, influencers, redes sociales, estudiantes

\section{Introducción:}

El fenómeno de la influencia está presente en la vida de las personas y resulta de gran importancia debido a que es capaz de cambiar actitudes y comportamientos en los mismos; es decir fruto de la interacción entre las personas, surgen los líderes de opinión, personas que poseen una serie de características, entre ellas la credibilidad, y que son capaces de ejercer una influencia directa sobre un colectivo.

Las marcas a través de la publicidad han querido acercarse a sus públicos otorgándole credibilidad a sus productos. Buscando esa credibilidad, entre otras características, y teniendo en cuenta la importancia de la influencia entre los individuos, han surgido en el mundo publicitario los llamados prescriptores.

A través del paso del tiempo y el avance de la tecnología, se ha ido incrementando el número de prescriptores en la sociedad y han traspasado los medios convencionales. Los prescriptores han evolucionado y llegado al panorama 2.0, en el que cualquier persona puede convertirse en uno, naciendo así los denominados influencers. En la actualidad han obtenido gran notoriedad debido al gran auge que han vivido en un corto periodo de tiempo y la importancia que han obtenido para las empresas, ya que con los recursos con los que se cuentan hoy (las plataformas sociales) se puede llegar a un gran número de individuos.

Estas plataformas sociales juegan un papel fundamental en cuanto a la influencia en la formación de los estudiantes de la facultad de ciencias, han cobrado tal importancia en los últimos años que según un estudio realizado en 2015 por la empresa japonesa Takumi, se encuentran entre los 15 agentes que más influyen en el comportamiento del consumidor (Cfr. Stewart, 2015). En la presente ponencia el objetivo a alcanzar radica en el análisis del impacto de los influencers en los estudiantes de la Facultad de Administración de Ciencias Empresariales de la Universidad Técnica Estatal de Quevedo. 


\section{Metodología:}

El desarrollo de la presente investigación se fundamenta, en métodos de nivel teórico, empírico, como: análisis y síntesis, cualitativo, histórico-lógico, lo cual implica una profunda revisión bibliográfica para abstraer el fundamento teórico a la realidad actual.

\section{Resultados:}

"Las tecnologías cambian, la gente cambia, los usuarios cambian. Tienes que adaptarte o serás como una cinta de cassette en la era, no ya de los CD sino del mp3." Seth Porges

La transformación digital en la que actualmente nos encontramos ha generado que los procesos cotidianos de comunicarnos e interactuar cambien, para desarrollar nuevas alternativas rápidas y novedosas que nos acercan a múltiples personas en segundos y nos facilite realizar tareas tan sencillas como comprar, hablar, conocer y compartir información.

Sin darnos cuenta la nueva era tecnológica nos ha hecho adaptarnos a un nuevo modelo de comunicación y de información, para Marshall McLuhan (citado por Ariel, 2011) "somos lo que vemos, formamos nuestras herramientas y luego éstas nos forman". Se cree que los medios son fuentes a través de las que recibimos información, pero la concepción de McLuhan explicaba que cualquier tecnología es una extensión de nuestro cuerpo, mente o ser. De esta manera, los medios tecnológicos son entendidos como herramientas que extienden las habilidades humanas, así como una bicicleta o un automóvil es una extensión de nuestros pies (Ariel, 2011). Los medios de comunicación son una extensión de los sentidos o capacidad psíquica del hombre.

Del mismo modo (McLuhan citado por Ariel 2011) afirma que el medio es el mensaje, y la tecnología modifica al hombre, ya que el medio de comunicación influye, determina y moldea.

Los autores consideran que todos los medios nos mueven con fuerza y nos transforman completamente. El medio es el mensaje, ya que este ha impulsado los cambios sociales y culturales, que, de igual forma, solo pueden ser comprendidos si se conoce la manera en que los medios se funcionan en el ambiente. Toda tecnología tiende a crear un nuevo medio ambiente o "galaxia" que no funciona como un recipiente pasivo, sino por el contrario, opera como un proceso activo que da nueva forma tanto al hombre como a otras tecnologías.

Por otro lado, Manuel Castells (2009) afirma que comunicar es compartir significados mediante el intercambio de información. "El proceso de comunicación se define por la tecnología de la comunicación, las características de los emisores y los receptores de la información, sus códigos culturales de referencia, sus protocolos de comunicación y el 
alcance del proceso" (p. 87). Con la difusión de internet, se da una nueva forma de comunicación interactiva caracterizada por la capacidad de enviar mensajes de muchos a muchos, en tiempo real o en un momento concreto.

"Existe una transformación tecnológica basada en la digitalización de la comunicación, la interconexión de ordenadores, el software avanzado, la mayor capacidad de transmisión por banda ancha y la omnipresente comunicación local-global por redes inalámbricas, de manera creciente con acceso a internet" (Castells citado por Castillo, 2017). Los medios son predominantemente un negocio, por lo que las mismas tendencias que han transformado el mundo empresarial, es decir que la globalización, digitalización, creación de redes y desregulación, han alterado sustancialmente las operaciones mediáticas.

Por esta razón, la sociedad tuvo que adaptarse a las nuevas tecnologías y sufrió una transformación en la manera de comer, consumir, vender e, incluso, vivir. Por todo lo anterior, los negocios también se vieron en la necesidad de replantear su manera de comunicarse con el público y el consumidor, a partir de la implementación de diferentes estrategias para dar a conocer su marca y de transformarse para ser competitivos, lograr cumplir las necesidades de las personas y enganchar al cliente con su marca. Por ello es importante conocer la marca y cómo funciona, para crear una comunicación más directa y adecuada y así lograr el objetivo que se desea que sea siempre conocer y comunicarse con el consumidor.

Así mismo, la marca se puede considerar como un concepto complejo debido a que se desarrollan procesos gráficos, visuales, psicológicos y culturales. Igualmente, no se puede centrar en una sola área de estudio dada a su capacidad de poder analítico desde una perspectiva, social, económica, artística y comunicativa.

El autor Joan Costa (1994, citado por Barroso, Gómez \& Guardia, s.f), afirma que la marca es un distintivo gráfico de identidad, una figura icónica que relaciona la idea con el objeto. Su destino primordial es un gran número de relaciones y agrupaciones mentales. También, supone que la capacidad asociativa de la marca es una condición importante, para que, con varios sentidos a la vez, se utilice como representación o figura icónica (signo).

Por su parte, Philip Kotler considera que "ya sea que se trate de un nombre, una marca comercial, un logotipo u otro símbolo, una marca es en esencia la promesa de una parte vendedora de proporcionar, de forma consistente a los compradores, un conjunto específico de características, beneficios y servicios" (Kotler, 2001).

De esta manera, la marca es una denominación o referente, un conjunto de valores, un estatus o una condición y un sin fin de cualidades. Estas cualidades permiten que se pueda ver cómo marcas de reconocido prestigio son deseadas por la sociedad, mientras otras quedan relegadas a un segundo plano. Por ello, el objetivo de una marca es producir 
una serie de estímulos positivos que la posicionan de tal manera que favorezca su consumo y su percepción por parte de la sociedad.

Pero sin importar la definición de dicha marca, todas buscan lograr un posicionamiento en el mercado y apropiarse del corazón y el dinero de los consumidores por eso, como lo afirma Kotler (1973, citado por Barrios, 2012), las marcas tienen que posicionarse de manera diferente y no solo a través del precio o el surtido. Al contrario, estas deben aprovechar este mundo virtual para conocer el comportamiento de los clientes y crear un entorno de consumo que aumente las posibilidades de compra a través de la experiencia.

Por eso, las empresas incursionan en las redes sociales para tener un canal bidireccional de comunicación con sus clientes, para saber que les interesa y qué aspectos los insatisface frente al producto o servicio que ofrecen. Estas herramientas de escucha han permitido que ya no se hable a un grupo general de personas, sino que los mensajes sean realizados para comunidades específicas y se capturen, de manera más efectiva, a clientes potenciales. Según Castelló el surgimiento de las redes sociales y de los entornos 2.0 han ofrecido a los usuarios la posibilidad de no sólo compartir sus opiniones y experiencias sino contar con un rol de productores y consumidores (2014).

Es necesario destacar como las redes sociales se consideran un servicio que se ofrece a través de una plataforma web que permite a las personas construir un perfil público o semipúblico dentro de un sistema delimitado, lo que permite una comunicación con otros usuarios con quien se quiere compartir e interactuar (Boyd y Ellison, 2007).

Pero el mundo digital no sólo ha permitido la interconexión sino se ha convertido en la manera más habitual para obtener información sobre cualquier tema de interés debido a que todo está en un click. Así mismo, como lo afirma Uribe (2010), la web generó una evolución en las formas de interacción, permitiendo una constante e inmediata comunicación: Internet y los cambios tecnológicos han transformado el mundo en las últimas décadas. La forma en que se comunican las personas ha cambiado. La aparición de redes sociales digitales (RSD) en Internet ha permitido que las personas puedan comunicarse de otra forma utilizando las nuevas tecnologías, generando relaciones que posibilitan la interacción constante e inmediata.

De tal manera, el principal cambio que trae consigo este nuevo mundo digital es la posibilidad de estar conectados en todo momento y en cualquier lugar (ANETCOM citado por Uribe, Riald, Llonc, 2013).

Debido a la influencia del mundo digital en los diversos espacios de la vida del ser humano, los procesos para entender el consumidor han cambiado, generando la necesidad de crear estrategias más asertivas de comunicación con el cliente o usuario. De tal forma, como lo afirma Cangas, el marketing digital en un inicio se desarrolló como un canal de promoción de productos o servicios que estuviera más cercano al consumidor y debido a la evolución tecnológica se ha convertido también en una herramienta para gestionar y 
analizar datos sobre sus consumidores (2010).

Se considera que el término de marketing digital se utilizó por primera vez en la década de 1990; sin embargo, debido a la incursión del internet y las redes sociales, este término ha tomado fuerza al convertirse en un medio para crear una relación directa y permanente con el consumidor.

Al ser una herramienta de gran utilidad, las empresas han decidió no desaprovechar la oportunidad de utilizar estos medios digitales para lograr alcanzar sus objetivos, dado que "el internet es una de las herramientas del marketing más eficaces que una compañía puede utilizar para promover su marca, su servicio o sus productos, ya que nos ofrece una completa gama de recursos que le permite a las empresas llegar a los clientes tanto antiguos como recientes" (Collin, 2003, citado por Montero, 2015).

El avance del marketing digital ha proporcionado a las empresas "la oportunidad de crear valor para los clientes y construir fuertes relaciones con ellos con el propósito de obtener a cambio valor procedente de dichos clientes" (Kotler, 2008). Por eso, el objetivo de esta área de estudio en las ciencias empresariales es hacer que los clientes se interesen, soliciten el producto o servicio que se ofrece y, finalmente, se conviertan en clientes fidelizados con la marca.

De esta manera, como lo afirma Castelló (2014), los espacios digitales se han convertido en la mejor publicidad para las empresas, motivo por el que se genera la necesidad de crear una buena reputación digital y gestionar una conversación en estos entornos virtuales que atienda a las críticas para conservar la confianza de sus clientes actuales y potenciales.

Sin embargo, para obtener los resultados esperados en los entornos digitales es necesario lograr un posicionamiento de marca por medio del acercamiento con el consumidor. Esto se logra a través de una estrategia comunicativa donde se entienda al cliente desde sus esferas más pequeñas de interrelación con su entorno.

Con el fin de acercar a las marcas a los usuarios por medio de las redes digitales, se vuelve indispensable el concepto de confianza. Según cifras de 2015 de Global trust in Advertising Report", el $83 \%$ de los consumidores confian plenamente en las recomendaciones de amigos y familias, y $66 \%$ dice que confía en las opiniones que otros usuarios postean online. Por esto, los influencers se han convertido en una valiosa opción para las compañías. El secreto de los influenciadores es su cercanía a los usuarios, ya que, a medida que comparten a diario contenido atractivo que incluso hace parte de su vida personal, atraen a un público que puede llegar a sentir al influencer como un "amigo" o "conocido". A partir de este enlace emocional, el público sigue sus pasos de manera casi incondicional y por este motivo algunas marcas deciden unirse y aprovechar esta confianza para desarrollar una nueva estrategia para posicionarse en el mercado.

El marketing de influencers según Geno Church, comienza por entender al ser humano, 
su nuevo entorno y su comportamiento sobre él mismo (Citado por Anzures, 2016). De esta manera, se puede entender que:

"La inclusión digital como youtubers que son individuos, que utilizan la red social de vídeos más grande del mercado digital YouTube para postear cualquier tipo de contenido que pueda resultar entretenido, generando así un gran número de reproducciones, snapchaters, aquellas personas que usan frecuentemente la aplicación de Snapchat y tienen un gran número de seguidores, periscopers, personas con un gran número de seguidores y de visualizaciones cuando realizan un video en vivo por medio de la aplicación Periscope y askers, que proviene de la palabra Ask, la cual es una red social de preguntas y respuestas, han desarrollado sus propios contenidos y cada uno de ellos ha creado a partir de esto sus propias comunidades y seguidores, lo que indica que en el futuro estas comunidades influenciarán a otras personas en consecuencia.

Así que tenemos que entender los nuevos modelos y por supuesto a las personas detrás de ellos, porque comprendiendo los comportamientos humanos, podremos desarrollar mejores herramientas tecnológicas”. (Citado por Anzures, 2016).

Por poner un ejemplo, algunos influencers deciden enfocarse en un tema específico a la hora de crear los contenidos que compartirán en la red, entre los temas en que se suelen especializar es la gastronomía. La alimentación es una de las necesidades fisiológicas más importantes del hombre y además una de las áreas con más demanda en el mercado, por tal razón, algunos influencers buscan implementar estrategias creativas para el desarrollo del marketing gastronómico, que surge como una alternativa para ayudar a los restaurantes a diseñar un mejor posicionamiento en el mercado, con el objetivo de elaborar una oferta atractiva, accesible y vinculada al mercado actual.

Cabe recordar que para Philip Kotler, uno de los principales autores en la materia, "el marketing es un proceso social y administrativo mediante el cual grupos e individuos obtienen lo que necesitan y desean a través de generar, ofrecer e intercambiar productos de valor con sus semejantes" (2001, p. 7).

Entonces, la función del marketing es precisamente educar al mercado, es decir, a los consumidores, antes, durante y después de entrar en contacto con el proveedor. El mercadeo consiste en "vender" ideas, no se trata sólo de una relación transaccional de trasladar dinero de una mano a otra. El hecho de que diversas acciones de marketing conlleven, en algunos casos, a un intercambio económico no implica que esta sea la esencia del marketing.

Las páginas web y redes sociales son herramientas clave en la estrategia comunicativa, no sólo por el ejercicio corporativo que en ellas se observa y el trabajo de imagen que se puede apreciar, sino también por la existencia evidente de recursos web que contribuyen al fomento de la comunicación bidireccional.

Además, en la formación de los estudiantes en la Facultad de Ciencias Empresariales de 
la Universidad Técnica Estatal de Quevedo se debe tener en cuenta que para emplear las estrategias de marketing y de influencers es necesario tener claro la razón de ser de la marca, y el público objetivo al que se piensa dirigir para así tener un alcance efectivo.

Sin lugar a dudas, esta evolución digital transformó todos los espacios comunicativos ya establecidos y permitió desarrollar nuevas maneras de acercarse y entender el consumidor en sus mundos particulares. Por ejemplo, esos argumentos han permitido en la actualidad, que los restaurantes busquen ese punto diferenciador en sus propuestas gastronómicas y ambientes parar brindar una experiencia completa al cliente, para que ellos mismos puedan generar un eco en su círculo de allegados.

El estudiante de la Facultad de Ciencias Empresariales de la Universidad Técnica Estatal de Quevedo debe profundizar en el conocimiento de sobre los influencers en su contexto. Para ello, es fundamental de lo anteriormente explicado, entender que los 'Influencers' son "personas que tienen gran poder de influencia en los medios de comunicación, con un gran número de seguidores en sus cuentas de redes sociales y con un gran prestigio" (Carricajo, 2015, pág. 19). Sin embargo, es válido afirmar que cualquier persona que desee ser un influenciador en las redes sociales, lo puede hacer simplemente creando un perfil en una red social.

La siguiente suposición explicará mejor lo anterior mencionado. Sí se está navegando en una red social y se encuentra con un usuario o una página de ' $\mathrm{X}$ ' marca que llama la atención, es muy probable que como usuario entre y la 'stalkee' (investigar perfiles o páginas de otros usuarios) un poco para conocer de ella; seguido a esto, ese usuario se entera que esta persona o marca cuenta con muchos amigos o seguidores, y diariamente recibe muchos 'likes' en todas sus fotos, videos, publicaciones, etc. (...) Hasta la información suministrada no es de mucha relevancia, sin embargo, éste usuario observa que en una de sus fotos ésta persona tiene una camiseta que puede interesarle (la camiseta vista como producto), puesto que el estilo y otros factores le llaman la atención.

Al parecer los datos suministrados no dicen mucho de la persona, o si ésta pretende convencer a algún otro usuario; empero, el usuario que está observando la foto se da cuenta que hay un comentario por parte de esa persona diciendo el lugar y posiblemente el precio de esa prenda de vestir. Por ende, si dicho usuario decide hacer la compra de tal producto, estaría, posiblemente sin saber, siendo influenciado por ese desconocido o marca desconocida que encontró en la red social y ciertamente, este usuario continuará visitando ese perfil en un futuro próximo.

Esto refleja que las primeras influencias que los seres humanos tenían por parte de su familia, amigos y gente cercana, comenzó a expandirse gracias a la Web 2.0, hasta el punto de 'convertirse en retazos' de personas, llamadas 'Influencers', que, con publicaciones en las redes sociales, influyen en los estilos y comportamientos de vida de otros usuarios. Cory Kennedy, reconocida 'Influencer' que lleva varios años en este medio, afirmó que nunca llego a creer que el contenido que publicaba sería tan relevante 
para otras personas que no conocía (Sisí, 2015). Sin embargo, no todo es color de rosa; ser 'Influencer' es vivir en un mundo de estereotipos perfectos impuestos por la sociedad, llega un momento en que "a pesar de ser maravilloso el hecho de tener tantos seguidores y de seguir online en todas las plataformas populares, ya no siente lo mismo que al ser una pionera en tierras digitales" (Sisí, 2015).

Es importante resaltar que los 'Influencers' son herramientas que son capaces de crear tendencias en los consumidores e influir en las decisiones de consumo de los mismos, sirviendo como apoyo a las marcas que los utilizan como canales de difusión y distribución (Gillin, 2009). Esto deja claro, que este fenómeno se ha convertido en una de las herramientas más fuertes que utiliza el marketing para la implementación de sus estrategias de comunicación.

Los estudiantes de la Facultad de Ciencias Empresariales de la Universidad Técnica Estatal de Quevedo deben tener claro que estas herramientas influenciadoras son utilizadas para la promoción de marcas y todo lo que ellas puedan ofrecer al público, pero, también son necesarias "a la hora de superar una crisis, las empresas suelen tener en cuenta a los Influencers como una herramienta de marketing más económica e instantánea" (Nocito, de Moya, Gutiérrez, \& Rothe, 2017, pág. 8). Este es uno de los factores por el cual los 'Millennials' ven este camino como la mejor opción para trabajar y tener un mejor estilo de vida, sin embargo, llegar a este punto no es tarea fácil. Debe existir una estrategia, además de la persistencia por parte del gestor y la originalidad de sus contenidos.

No existe un manual que explique al estudiante de la Facultad de Ciencias Empresariales de la Universidad Técnica Estatal de Quevedo cómo se debe comenzar para ser un influenciador en la web, sin embargo, Ciancio (2017), explica que es necesario hacer una lista de las pasiones para elegir cuál es aquella que mejor se maneja, conoce a fondo y puede conversar durante horas sin titubear. Es necesario elegir un "tema en el cual más se destaque para que su público busque su aporte, ya sea en matemática, moda, música, o medicina. Es fundamental que se defina a quiénes dirigirse y se adapte su vocabulario a esa audiencia específica" (Ciancio, 2017).

Es válido afirmar que lo primero que se debe definir es el tema al cual se va a enfocar la marca personal (Influencer), para establecer el tipo de 'Influencer'.

Tipos de Influencers.

Según Marketing Directo (2016), los 'Influencers' son importantes para las marcas, sin embargo, no todos funcionan para una misma marca. Por tal motivo se realizó una diferenciación y dio como resultado 6 categorías, ésta sería la definición de cada uno según Marketing Directo (2016):

\section{EL HEALTHY}

La mayor conciencia social sobre la importancia no solo de llevar una buena alimentación 
o realizar ejercicio físico, sino también de mantener un estilo de vida beneficioso en todos los ámbitos, ha dado lugar a este tipo de Influencers.

\section{EL COLABORATIVO}

El entretenimiento o la comedia ya no son cosa de una sola persona. Cada vez es más frecuente ver a los Youtubers juntarse para colaborar en la creación de nuevos vídeos y aglutinar seguidores en torno a sus canales.

\section{EL ESTILOSO}

Estas personalidades se han convertido en iconos de la moda añadiendo su personalidad y estilo propios más allá de las marcas.

\section{EL GAMER}

Los videojuegos aglutinan a un público muy característico y, sobre todo, muy fiel. Es por ello que este campo resulta un mercado muy atractivo para las marcas que quieran lograr el engagement de los "gamers" y por el que Tinder o Red Bull ya apuestan.

\section{EL COCINERO}

La gastronomía y la cocina ha adquirido una nueva percepción acercando el placer culinario al público más joven.

\section{EL AVENTURERO}

Los Influencers que se agrupan en esta categoría, como Jackson Harries, convierten sus andanzas por el mundo en auténticas historias y narrativas que han enganchado a 1,8 millones de usuarios durante 3 años.

Influencer ¡has lo tuyo!

Entendiendo al 'Influencer' como una herramienta para la publicidad y construcción de una marca (Branding), es momento de indagar como se debe utilizar y en qué momentos es pertinente sacar este as bajo la manga.

La mayoría de marcas le están apostando es estas herramientas, a veces sin conocer su potencial o funcionamiento. El 'Influencer marketing', como también se le conoce a esta práctica, es una de las tendencias más fuertes de la actualidad, "no es para menos pues, la naturalidad, visibilidad y credibilidad que las nuevas estrellas de la red añaden al discurso de las compañías es garantía de éxito" (Marketing Directo, 2016).

Según Muela y Baladrón (2010) “el consumidor 2.0 se informa mucho antes de realizar una compra y, por tanto, ésta es mucho más racional”. Puesto que este tipo de consumidor ya no cree en la falsa realidad que la publicidad genera, fue necesario que se demostrara la realidad con personas reales, conocidos ahora como 'Influencers'.

Lo que hace el 'Influencer marketing' es transformar la visión de las cosas, mostrar una realidad con realidad, cambiando por completo el modelo tradicional de marketing que la mayoría de marcas había utilizado. Los 'Influencers' ofrecen sus opiniones 
personalizadas, sabiendo que cuentan con la confianza de sus consumidores, es decir, Target; este les da la libertad de no ser imparciales, por lo tanto, sus dichos juicios se toman con realidad, como el consejo que un conocido da (Díaz, 2017).

La confianza que estos personajes adquieren por parte de sus consumidores, crea un vínculo, una relación emocional que es proyectada por un personaje del común; es decir, el público lo ve como alguien normal que tiene una voz en el mundo. Aquí es posible observar una de las estrategias que el Branding Emocional utiliza para llegar al cliente efectivamente.

Las nuevas generaciones de consumidores son cada vez más difíciles de convencer, no solo porque no confían en la publicidad, también pretenden y quieren ser únicos. Se debe tener en cuenta que este tipo de personajes, refiriéndonos a los nuevos consumidores, toman decisiones por lo que ellos creen y piensan (la emoción sobre la razón), siempre "será más fácil trabajar sobre las percepciones o prejuiciosos que tienen los consumidores, que cambiar esas percepciones preconcebidas" (Chen, 2014).

La clave de éste es identidad, identificación con el otro, él (Influencer) es el público (Consumidor), él los entiende y sabe lo que quieren.

Entendiendo la identificación como la clave del éxito; una marca debe conocer primero su Target, a quién quiere dirigirse y vender su producto o servicio. Después de ello, elegir cuáles serían los mejores canales de difusión para alcanzar el objetivo propuesto; el paso más lógico consiste en buscar cuál sería la categoría de 'Influencers' que necesita y cuál de todos ellos es el más adecuado. Por lo tanto, el estudio de los 'Influencers constituye una de los contenidos fundamentales en la formación de estudiantes de la Facultad de Ciencias Empresariales de la Universidad Técnica Estatal de Quevedo

Ellos, a través de la red, apoyan a diferentes marcas, y las promocionan mediante su contenido multimedia, que llega a millones de personas (sus seguidores o followers)" (Nocito et al., 2017, pág. 28).

Es claro que "el poder de recomendación e influencia gracias a las redes sociales es muy efectivo, y este hecho no ha pasado desapercibido para las marcas y empresas" (Castelló y del Pino, 2015, pág. 47). Además, Castelló y del Pino (2015) aseveran que, las acciones publicitarias con influenciadores digitales reproducen la figura del tradicional prescriptor en publicidad, pero con particularidades propias de un nuevo marketing, que pone de manifiesto los cambios ante los que nos encontramos, producidos al hilo de la actual revolución tecnológica.

Lo cual deja claro que en el mundo transmedia y multidisciplinar en el que los consumidores viven actualmente, los 'Influencers' se convierten en parte integral de la construcción de marca con la ayuda de la emoción (Branding emocional). 


\section{Conclusiones:}

- Influencer es una persona que cuenta con un destacado prestigio en redes sociales, este individuo debe contar con un numeroso grupo de fans que siguen sus publicaciones, éstas lo convierten en un prescriptor importante de información, opinión o de marca. Su misión es transformar la visión de las cosas cambiando por completo los modelos tradicionales de publicidad.

- Los estudiantes de la Facultad de Ciencias Empresariales de la Universidad Técnica Estatal de Quevedo deben adoptar un conocimiento absoluto de este contenido para ejercer con mayor calidad y competencias su gestión comercial, teniendo en consideración que los Influencers son personas que influyen en la parte emocional y los estilos de vida de un público a través de diversos espacios publicitarios con el fin de lograr el buen posicionamiento de una marca entre los consumidores.

- Los Influencers constituyen una salida publicitaria prometedora, de ahí su principal impacto en la formación de los estudiantes de la Facultad de Ciencias Empresariales de la Universidad Técnica Estatal de Quevedo, ya que son personas reales, del diario vivir, comunes y corrientes y con los cuales el público se siente identificado, son la novedosa oportunidad que aprovechan las empresas para lograr un impacto positivo sobre las marcas de forma inmediata en una gran cantidad de usuarios, son notoriamente buscados por empresas y organizaciones para que ayuden a recomendar el producto o servicio porque la publicidad tradicional no ha ejercido el impacto necesario para construir y posicionar una marca.

- Los Influencers a través de diversos medios o canales se acercan a los consumidores y por su conocimiento concreto del producto, han logrado impactar al usuario, le ha creado la necesidad de comprar y recordar la marca.

- Las estrategias son acciones que están encaminadas a lograr objetivos empresariales en publicidad, las estrategias ayudan a la organización al alcance de su misión a partir del diseño de un plan de acción.

- Son ocho los tipos de estrategias publicitarias: competitivas, financieras, comparativas, de posicionamiento, promocional, de empuje, de tracción y de imitación. Cada una de ellas aporta elementos importantes que le permiten al estudiante de la Facultad de Ciencias Empresariales de la Universidad Técnica Estatal de Quevedo en la organización maximizar el número de impactos en el público consumidor de tal forma que aumenta el volumen de ventas. No obstante, es necesario que la empresa u organización analice que tipo de estrategia es la más adecuada para lograr sus fines y propósitos. 


\section{Referencias Bibliográficas}

Anzures, F. (2016). Social Marketing influence. Liquid Thinking.

Barroso, C. Gómez,P. Guardia, M. (S,f). La marca en la hipermedia. Revista creatividad y sociedad.

Ariel, C. (2011). Nociones de cibercultura y periodismo.

Barrios, M. (2012). Marketing de la Experiencia: principales conceptos y características. Palermo Business Review.

Boyd, D., \& Ellison, N. (2007). "Social Network Sites: Definition, History, and Scholarship", Journal of computer-mediated communication. Journal of computer- mediated communication, 13(1). Recuperado de: http://onlinelibrary.wiley.com/doi/10.1111/j.10836101.2007.00393.x/full

Cabrera, S. (2013). Marketing gastronómico. La experiencia de convertir el momento del consumo en un recuerdo memorable. Recuperado de: http://www.scielo.org.ar/img/revistas/ccedce/n45/html/n45a15.htm

Carricajo, C. (2015). Marketing de influencers: una nueva estrategia publicitaria.

Universidad de Valladolid, España, Segovia. Tomado de: https://uvadoc.uva.es/bitstream/10324/13095/1/TFG-N.241.pdf

Castelló, A. (2014). La agencia de medios en el nuevo escenario comunicativo Media agency in the new communicative scenario. Revista Mediterranea. Recuperado de: https://dialnet.unirioja.es/descarga/articulo/4897644.pdf

Castello, A. \& Del Pino, C. (2015). La comunicación publicitaria con influencers. Revista Digital de Marketing Aplicado, 1 (14), p. 21-50.

Castells, M. (2012). Comunicación en la era digital. Recuperado de: http://masnuelcastells.blogspot.com.co

Castillo, M. (2017). Miedo, control social y política criminal. Madrid: Dykinson.

Collin, S. (2003). Estrategias de Mercadotecnia Para promover su marca en Internet. McGrawhill.

García o, M. (1993). La Encuesta. En M. García Ferrando, J. Ibáñez y F. Alvira (Comp.), El análisis de la realidad social. Métodos y técnicas de investigación (pp. 123-152). Madrid, España: Alianza Universidad

Gómez, M. (2006). Introducción a la metodología de la investigación científica. Argentina: Editorial Brujas.

Kotler, P. (2002). Dirección de marketing: Concepto esenciales. Pearson education. México.

Kotler, P. (2008). Fundamentos de marketing. México: Pearson Education.

Montero, L. (2015). Marketing Digital Como Mecanismo Para Optimizar Las Ventas En pymes del Sector Comercio En Colombia (Seminario de especialización). Universidad Militar Nueva Granada, Bogotá, Colombia.

Ramos, M. (2015) ... Instagramers Y Bloggers En La Comunicación De Moda Del Siglo XXI En España. Universidad de Valladolid, Segovía, España.

Revista Dinero. (2016, 15 de diciembre). La radiografía del mercado de comidas rápidas 
en Colombia. Revista Dinero.

Uribe (2010). Uso de las redes sociales digitales como herramienta de marketing - un estudio de casos. Universidad Autónoma de Barcelona. 


\section{PARA CITAR EL ARTÍCULO INDEXADO.}

Fonseca Vasconez, J. F., Ballesteros Ballesteros , E. Y., \& Vinueza Peralta, H. A. (2021). Impacto de los influencer en los estudiantes de la Facultad de Ciencias Empresariales de la Universidad Técnica Estatal de Quevedo. ConcienciaDigital, 4(1.1), 279-293. https://doi.org/10.33262/concienciadigital.v4i1.1.1559

\section{Ciencia \\ LDigital}

El artículo que se publica es de exclusiva responsabilidad de los autores y no necesariamente reflejan el pensamiento de la Revista Conciencia Digital.

El artículo queda en propiedad de la revista y, por tanto, su publicación parcial y/o total en otro medio tiene que ser autorizado por el director de la Revista Conciencia Digital.

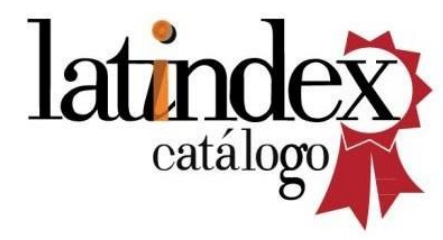

